\title{
花生 $\Delta^{9}$-硬脂酰-ACP 脱氢酶基因 $(S A D)$ 的序列分析
}

东金玉 万勇善 ${ }^{*}$ 刘风珍*

山东农业大学农学院 / 作物生物学国家重点实验室 / 山东省作物生物学重点实验室, 山东泰安 271018

摘 要: 利用同源克隆技术获得花生区组二倍体野生种 A. duranensis 和 A. ipaensis 的 $\Delta^{9}$-硬脂酰-ACP 脱氢酶基因 $S A D$ (命名为 $g S A D-A$ 和 $g S A D-B$ )及 3 个栽培品种的 $S A D$, 每个栽培品种有 2 个 $S A D$ (命名为 $g S A D-1$ 和 $g S A D-2$ )。同 时获得丰花 2 号 $S A D$ 的两条全长 cDNA (命名为 FhrSAD-1 和 FhrSAD-2)。丰花 2 号的 FhgSAD-1 和 FhgSAD-2 均含 有 2 个内含子, 二者同源性 $97.5 \%$, 共有 69 个变异位点, 其中 62 个是 SNP 位点、6 个特异性酶切位点。FhrSAD-1 和 FhrSAD-2 间核苷酸序列同源性 98.6\%, 其中编码区序列同源性 98.9\%, 共有 12 个变异位点, 编码的 Ah-SAD2 氨 基酸序列与 Ah-SAD1 相比在 $N$ 端的 ${ }^{17} \mathrm{PSSSSSSSSSSFSL}^{30}$ 丝氨酸聚集区少一个丝氨酸。gSAD-1 和 $g S A D-A$ 同源性为 $99.9 \%$, 存在 4 个 SNP 位点; $g S A D-2$ 和 $g S A D-B$ 同源性为 $100 \%$ 。推测 $g S A D-1$ 和 $g S A D-2$ 分别来自花生栽培品种的 A、 B 2 个染色体组。研究明确了花生不同染色体组 $S A D$ 的序列特征, 为进一步探讨 $S A D$ 的表达及其在控制花生籽仁脂 肪酸组分中的作用提供了重要的参考。

关键词：花生; 硬脂酰-ACP 脱氢酶(SAD); 序列分析

\section{Sequence Analysis of $\Delta^{9}$-Stearoyl-ACP Desaturase Gene (SAD) in Peanut}

DONG Jin-Yu, WAN Yong-Shan ${ }^{*}$, and LIU Feng-Zhen ${ }^{*}$

State Key Laboratory of Crop Biology / Shandong Key Laboratory of Crop Biology / Agronomy College of Shandong Agricultural University, Tai' an 271018, China

\begin{abstract}
The peanut (Arachis hypogaea L.) cultivars are an allotetraploid consisting of A and B genomes. $\Delta^{9}$-Stearoyl-ACP desaturase (SAD) is a key enzyme that catalyzes the conversion of stearoyl-ACP to oleoyl-ACP, and finally controls the content of oleic acid as well as the proportion of saturated to unsaturated fatty acids. By using the primers based on the peanut cDNA sequence of SAD (AF172728), genomic DNAs were amplified from the wild diploid species A. duranensis and A. ipaensis, and from the cultivated accessions of peanut Fenghua 2, Shanhua 7 and Puyangdatuoyang, respectively. Two isoforms of the genomic $S A D$ were identified and named as FhgSAD-1 and FhgSAD-2 from the cultivated peanut accessions. In addition, two isoforms of $S A D$ cDNA were isolated and named as FhrSAD-1 and FhrSAD-2 from Fenghua 2. Comparison of the genomic sequences with cDNAs revealed that there were two introns in the $S A D$ genomic sequences. Sequences alignment showed that the similarity between FhgSAD-1 and FhgSAD-2 in nucleotide level was over 97.5\%, with 69 different sites in total, including 62 SNP sites and six variation sites of endonuclease recognition. The cDNA sequence similarity between FhrSAD-1 and FhrSAD-2 was $98.6 \%$, with $98.9 \%$ nucleotides identity in coding region. Deduced amino acid sequences revealed that only one difference occurred in serine gathering area of ${ }^{17} \mathrm{PSSSSSSSSSSFSL}^{30}$. gSAD-1 shared $99.9 \%$ nucleotide sequence homology with $g S A D-A$, while $g S A D-2$ was the same as $g S A D-B$. According to the phylogenetic tree, it is assumed that $g S A D-1$ and $g S A D-2$ may come from the $\mathrm{A}$ and $\mathrm{B}$ genome, respectively. The results revealed the characteristics of $S A D$ sequences from different genomes of peanut, and provided important basis for exploring gene expression regulation and fatty acid component improvement in peanut seeds.
\end{abstract}

Keywords: Peanut; Stearoyl-ACP desaturase (SAD); Sequence analysis

花生是我国重要的油料和经济作物之一, 总产 居世界首位。花生籽仁咜藏油脂中 $80 \%$ 左右为不饱
和脂肪酸, 饱和脂肪酸占 $20 \%$ 左右 ${ }^{[1-2]}$ 。植物不饱和 脂肪酸组成很大程度上是受到脂肪酸脱氢酶种类和

\footnotetext{
本研究由山东省花生良种产业化工程项目和现代农业产业技术体系建设项目(CAIS-14)资助。

* 通讯作者(Corresponding authors): 万勇善, E-mail: yswan@sdau.edu.cn, Tel: 0538-8241540; 刘风珍, E-mail: liufz@sdau.edu.cn, Tel: 0538-8241540

第一作者联系方式: E-mail: dongjinyu86@163.com

Received(收稿日期): 2011-12-29; Accepted(接受日期): 2012-04-15; Published online(网络出版日期): 2012-05-11.

URL: http://www.cnki.net/kcms/detail/11.1809.S.20120511.1540.020.html
} 
数量的调控, 而 $\Delta^{9}$-硬脂酰-ACP 脱氢酶(stearoylACP desaturase, SAD)催化硬脂酰-ACP 脱饱和而在 脂肪酸链的 C9 与 $\mathrm{C} 10$ 间引入一个双键形成油酰- $\mathrm{ACP}$ 的反应, 是不饱和脂肪酸合成代谢的关键酶 ${ }^{[3-4]}$ 。因此, $\mathrm{SAD}$ 直接决定了植物油脂中的不饱和脂肪酸的总量 以及饱和脂肪酸与不饱和脂肪酸的比例 ${ }^{[5-6]}$ 。目前, 在大豆 ${ }^{[7-8]}$ 、拟南芥 ${ }^{[9]}$ 等很多植物中已经克隆到 $S A D^{[10-12]}$, 经研究证实在大多数高等植物中, $S A D$ 呈 现组织特异性表达, 且在发育的种子中的表达量比 其他组织都高 ${ }^{[9,13-15]}$ 。 Liu 等 ${ }^{[16]}$ 通过 RNA 干涉使棉 花的 SAD 沉默, 硬脂酸含量从正常的 $20 \%$ 上升到 $40 \%$, 同时还伴随棉籽油中其他 3 种主要脂肪酸棕 桐酸、油酸和亚油酸的减少。Wendy 等 ${ }^{[17]}$ 将土豆 $S A D$ 转入烟草, 发现叶片和种子的脂肪酸组分发生了变 化, 不饱和脂肪酸含量增加。Jung 等(2000 年)首先 在 GenBank 中注册了花生 $S A D$ 的全长 cDNA 序列 (AF172728), 关于栽培品种不同染色体组 $S A D$ 序列 特征的研究末见报道。

本研究目的是通过对花生 3 个栽培品种和 2 个 野生种 $S A D$ 的 cDNA 和基因组 DNA 序列结构分析, 比较 $\mathrm{A} 、 \mathrm{~B}$ 染色体组的 $S A D$ 核苷酸序列和编码的氨 基酸序列差异, 及花生与其他油料作物的系统发育 关系, 为进一步利用 $S A D$ 改善油料作物中脂肪酸组 分提供重要的参考。

\section{1 材料与方法}

\section{1 试验材料}

花生属花生区组二倍体野生种 A. duranensis 和

A. ipaensis 及栽培品种誉阳大拖秧由中国农业科学 院油料作物研究所姜慧芳研究员提供; 栽培品种丰 花 2 号、山花 7 号由山东农业大学选育。

TIANgel 胶回收试剂盒购自天根生化科技有限 公司; pEASY-T1 Vector, 大肠杆菌菌株 DH5a, Trizol Reagent Kit, EasyScript First-Strand cDNA Synthesis Kit 购自北京全式金生物技术有限公司，其他试剂 均为进口或国产分析纯。引物由上海生物工程有限 公司合成, 测序工作由北京六合华大基因科技有限 公司完成。

\subsection{DNA、RNA 提取及 cDNA 链的合成}

分别取花生栽培品种和野生种幼嫩花生叶片, 用 CTAB 法提取总 DNA。丰花 2 号花生果针入土约 $35 \mathrm{~d}$ 后, 取幼果, 液氮速冻, $-70^{\circ} \mathrm{C}$ 保存, 参照 Trizol Reagent Kit 说明书提取其总 RNA。以 $2 \mu \mathrm{L}$ RNA 为模板、
以 Oligo $(\mathrm{dT})$ 为引物, 按照 EasyScript First-Strand cDNA Synthesis Kit 描述的方法，合成 cDNA 第一链。

\section{3 花生 $S A D$ 的 PCR 扩增}

采用报道的花生 $S A D$ 的 cDNA 序列(GenBank 登 录号为 AF172728)设计 1 对特异性引物, 上游引物 $\mathrm{F}$ : 5'-GCAACACGAAAAATGGCTCT-3'; 下游引物 R: 5'-GGACACCACACCGAAAACAT-3'。以花生栽培品 种丰花 2 号总 RNA 经反转录生成的 cDNA 第一链为 模板，用引物 $\mathrm{F}$ 和 $\mathrm{R}$ 扩增 $\mathrm{SAD}$ 的 cDNA 序列。PCR 程序为 $94^{\circ} \mathrm{C} 5 \mathrm{~min} ; 94^{\circ} \mathrm{C} 30 \mathrm{~s}, 58^{\circ} \mathrm{C} 30 \mathrm{~s}, 72^{\circ} \mathrm{C} 1.5 \mathrm{~min}$,

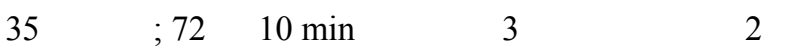
野生种基因组 DNA 为模板, 用引物 $\mathrm{F}$ 和 $\mathrm{R}$ 扩增 $S A D$ 的 DNA 序列。扩增程序为 $94^{\circ} \mathrm{C} 5 \mathrm{~min} ; 94^{\circ} \mathrm{C} 30 \mathrm{~s}$, $55^{\circ} \mathrm{C} 30 \mathrm{~s}, 72^{\circ} \mathrm{C} 3 \mathrm{~min}, 35$ 个循环; $72^{\circ} \mathrm{C} 10 \mathrm{~min}$ 。

\subsection{PCR 产物的克隆测序和序列分析}

PCR 扩增产物经 $10 \mathrm{~g} \mathrm{~L}^{-1}$ 琼脂糖凝胶电泳后, 按照 DNA 胶回收试剂盒说明书回收, 再与 pEASY$\mathrm{T} 1$ 克隆载体连接, 转化至大肠杆菌 DH5 $\alpha$ 中进行抗 卡那霉素篮选。挑选白色单菌落, PCR 检测鉴定后送 北京六合华大基因科技有限公司测序, 每个品种选 6 10 个克隆。采用 Blast 程序(http://www.ncbi.nlm. nih.gov/blast)分析序列的同源性, 用 DNAMAN 软件 进行序列翻译和多重比对。

\section{5 生物信息学分析}

利用 ClastalX 软件结合 PHYLIP 软件, 采用最 大简约法, 以来自栽培品种和野生种 $S A D$ 的 DNA 序列构建分子进化树。采用 BootStrap 1000 检验分 子系统树的置信度。利用软件 ClustalW 对包括花生 在内的 10 种主要油料作物 $S A D$ 编码的氨基酸序列 进行聚类分析。用下列在线软件对氨基酸序列进行 结构特征分析：蛋白等电点、分子量等分析软件 (http://www.expasy.ch/tools/protparam.html)，蛋白二 级结构分析软件 (http://npsa-pbil.ibcp.fr/cgi-bin/npsa automat.pl?page=/NPSA/npsa_sopma.html), 蛋白保守 结构域分析软件(http://www.ncbi.nlm.nih.gov/Structure/ cdd/wrpsb.cgi), 跨膜结构分析软件(http://www.cbs.dtu. $\mathrm{dk} /$ services/TMHMM/), 亚细胞定位软件(http://www. cbs.dtu.dk/services/TargetP), 信号肽预测软件 (http:// www.cbs.dtu.dk/services/SignalP).

\section{2 结果与分析}

\section{1 花生 $S A D$ 的克隆}

用引物 $\mathrm{F}$ 和 $\mathrm{R}$ 对丰花 2 号 $\mathrm{cDNA}$ 进行 PCR 扩 
增, 琼脂糖凝胶电泳检测获得约 $1.5 \mathrm{~kb}$ 特异性条带, 与预期大小相符。从琼脂糖凝胶回收的 PCR 产物与 pEASY-T1 克隆载体连接, 重组子转化大肠杆菌 DH $5 \alpha$ ，经卡那霉素篮选、PCR 检测鉴定获得含有目 的片段的阳性克隆, 测序结果表明, 存在 2 个 $S A D$ 基因, 分别命名为 FhrSAD-1 和 FhrSAD-2。

以 3 个栽培品种和 2 个野生种的 DNA 为模板, 用引物 $F$ 和 $R$ 对进行 PCR 扩增, 获得约 $3.5 \mathrm{~kb}$ 的片 段, 与 pEASY-T1 载体连接后, 克隆测序。结果表明, 3 个栽培品种均得到 2 条不同的序列, 命名为 $g S A D-1$ 和 $g S A D-2$ (表 1); 2 个野生种分别得到一条序列, 由 A. duranensis 克隆的序列命名为 $g S A D-A$, 由 $A$. ipaensis 克隆的序列命名为 $g S A D-B$ 。

\section{2 丰花 2 号 $S A D$ 核苷酸序列分析}

2.2.1 cDNA 序列分析 由丰花 2 号扩增得到的 cDNA 序列 FhrSAD-1 和 FhrSAD-2 同源性为 98.6\%, 与发表序列同源性分别为 $97.6 \%$ 和 $96.7 \%$ 。克隆得到 的两序列都具有开放阅读框, FhrSAD-1 全长 1479 $\mathrm{bp}$, 包含 $1221 \mathrm{bp}$ 的编码区和 $246 \mathrm{bp}$ 的 $3^{\prime} \mathrm{UTR}$; FhrSAD-2 全长 $1480 \mathrm{bp}$, 包含 $1218 \mathrm{bp}$ 的编码区和 $250 \mathrm{bp}$ 的 3'UTR; 起始密码子均位于 $13 \mathrm{bp}$ 处, 终止 密码子分别位于 $1231 \mathrm{bp}$ 和 $1228 \mathrm{bp}$ 处; ATG 起始 密码子附近符合 Kozak 规则(AXXATGG)(图 1)。 FhrSAD-1 和 FhrSAD-2 的编码区核甘酸序列同源性 $98.9 \%$, 共有 12 个变异位点, 其中 11 个为 SNP 位点 (图 1)。

表 1 供试的 3 个花生栽培品种及其对应的序列名

Table 1 Peanut cultivar names and the corresponding DNA sequences

\begin{tabular}{|c|c|c|c|}
\hline $\begin{array}{c}\text { 亚种名 } \\
\text { Subspecies }\end{array}$ & $\begin{array}{l}\text { 品种类型 } \\
\text { Variety type }\end{array}$ & $\begin{array}{c}\text { 品种名 } \\
\text { Accession }\end{array}$ & $\begin{array}{c}\text { DNA 序列名 } \\
\text { DNA sequence name }\end{array}$ \\
\hline \multirow[t]{2}{*}{ 疏枝亚种 ssp. fastigiata } & 珍珠豆型 var. vulgaris & 丰花 2 号 Fenghua 2 & FhgSAD-1, FhgSAD-2 \\
\hline & 中间型 Irregular type & 山花 7 号 Shanhua 7 & ShgSAD-1, ShgSAD-2 \\
\hline 密枝亚种 ssp. hypogaea & 普通型 var. hypogaea & 兴阳大拖秧 Puyangdatuoyang & PygSAD-1, PygSAD-2 \\
\hline
\end{tabular}

\subsubsection{DNA 序列分析 由丰花 2 号扩增得到的 2} 条 DNA 序列 FhgSAD-1 和 FhgSAD-2 长度分别为 $3503 \mathrm{bp}$ 和 $3510 \mathrm{bp}$, 同源性 $97.5 \%$ 。Splign 程序分 析表明, FhrSAD-1 和 FhrSAD-2 分别与 FhgSAD-1 和 $F h g S A D-2$ 外显子部分完全一致。FhgSAD-1 和 $F h g S A D-2$ 均含有 2 个内含子, FhgSAD-1 内含子位于 168 1 689、2 195 2 $695 \mathrm{bp}$ 处, 大小分别为 $1522 \mathrm{bp}$ 和 $501 \mathrm{bp} ; F h g S A D-2$ 内含子位于 165 1 695、2 201 $2699 \mathrm{bp}$ 处, 大小分别为 $1531 \mathrm{bp}$ 和 $499 \mathrm{bp}$ 。2 个序 列所有内含子的剪切方式均符合 GT-AG 规则(图 1)。

FhgSAD-1 和 FhgSAD-2 内含子核苷酸序列差异 分析表明, 2 个内含子分别存在 36 和 17 个差异位点, 其中 34 个和 14 个为 SNP (图 1)。全序列共有 69 个 变异位点, 其中 62 个 SNP, 占 $90.0 \%$ 。

用 DNAMAN 软件对序列分析发现, FhgSAD-1 具有 FhgSAD-2 没有的 Bsm I、Nde I、Apa I 和 Hind III 4 种限制性酶的识别位点, 而 FhgSAD-2 具有 FhgSAD-1 没有的 Age I 和 BstE II 两种限制性酶的识 别位点(图 2)。

\section{3 花生 SAD 核苷酸序列推导的氨基酸序列分} 析

由 FhrSAD-1 和 FhrSAD-2 推导出编码蛋白的氨
基酸序列, 分别命名为 Ah-SAD1 和 Ah-SAD2。二者 氨基酸序列同源性 $99.8 \%, \mathrm{Ah}-\mathrm{SAD} 1$ 全长 406 个氨基 酸, Ah-SAD2 全长 405 个氨基酸, Ah-SAD2 在 $\mathrm{N}$ 端 比 Ah-SAD1 少一个丝氨酸。将参考的该基因编码蛋 白的氨基酸序列命名为 Ah-SAD3, Ah-SAD3 全长 409 个氨基酸。Ah-SAD1 和 Ah-SAD3 序列同源性 $97.6 \%$ ，存在 9 个氨基酸差异(图 5)。

以 Ah-SAD1 和 Ah-SAD2 两条序列的生物信息 学分析预测 Ah-SAD1 的理论分子量和等电点, $\mathrm{MW}=46.229 \mathrm{kD}, \mathrm{pI}$ 6.43; Ah-SAD2 的理论分子量和 等电点为 $\mathrm{MW}=46.31 \mathrm{kD}, \mathrm{p} I 6.43$ 。二级结构分析表 明 Ah-SAD1 含 $50.62 \%$ 的 $\alpha$ 螺旋， $8.89 \%$ 的延伸串， $5.43 \%$ 的 $\beta$ 转角, $35.06 \%$ 的不规则卷曲; Ah-SAD2 含 $50.74 \%$ 的 $\alpha$ 螺旋, $9.16 \%$ 的延伸串, $5.45 \%$ 的 $\beta$ 转角, $34.65 \%$ 的不规则卷曲。Ah-SAD1 和 Ah-SAD2 蛋白 不存在明显的跨膜结构特征, 为可溶性蛋白。 Ah-SAD1 和 Ah-SAD2 定位于叶绿体的概率为 0.950 。

通过 NCBI 的 protein blast 功能、CD-Search 工 具及 $\mathrm{Cn} 3 \mathrm{D} 4.1$ 软件进行蛋白质保守结构分析表明: Ah-SAD1 和 Ah-SAD2 编码的多肽的保守性基本一 致，都是以同型二聚体形式存在，均含一个属于酰 
FhgSAD-1 GCAACACGAAAAATGGCTCTGAGGCTGAACCCTAACCCTTCACAGAAGCTCTTTCTCTCTCCTTCTTCAT

FhgSAD-2

FhgSAD-1

FhgSAD-2

FhgSAD-1

FhgSAD-2

FhgSAD-1

FhgSAD-2

FhgSAD-1

FhgSAD-2

FhgSAD-1

FhgSAD-2

FhgSAD-1

FhgSAD-2

FhgSAD-1

FhgSAD-1

FhgSAD-2

FhgSAD-1

FhgSAD-2

FhgSAD-1

FhgSAD-2

FhgSAD-1

FhgSAD-2

FhgSAD-1

FhgSAD-2

FhgSAD-1

FhgSAD-2

FhgSAD-1

FhgSAD-2

FhgSAD-1

FhgSAD-2

FhgSAD-1

FhgSAD-2

FhgSAD-1

FhgSAD-2

FhgSAD-1

FhgSAD-2

FhgSAD-1

FhgSAD-2

FhgSAD-1

FhgSAD-2

FhgSAD-1

FhgSAD-2

FhgSAD-1

FhgSAD-2

FhgSAD-1

FhgSAD-2

FhgSAD-1

FhgSAD-2

FhgSAD-1

FhgSAD-2

FhgSAD-1

FhgSAD-2

FhgSAD-1

FhgSAD-2

FhgSAD-1

FhgSAD-2

FhgSAD-1

FhgSAD-2

FhgSAD-1

FhgSAD-2

FhgSAD-1

FhgSAD-2

FhgSAD-1

FhgSAD-2

FhgSAD-1
CATCATCTTCTTCTTCTTCATCGTTCTCGCTTCCTCAAATGGCTAGCCTCAGATCTCCAAGGTTCCGCAT

GGCCTCCACCCTCCGCACTGGTTCCAAGTCTGTTCCTTTTTCCTTTCTCTCTTTCTCCCTTCСATTCCTC

GTCGATTGCTTTTCCCGATTTTAATTTCCTGAAATCAATGCCAAGACGCTGAAACCACGCTGA

CTCGATCTGCTTTTTCCCGATTTTTAATTTCCTGAAATCAATGCCAAGCACGCTGAAAACCACGCCTGAT

TITTCATTCACGTTTTTAATTITTGTTCTATAATGCTGTTTATTCATTTAATTACTTCTGTTGAATACTG

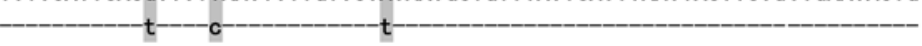

TGTTATGTGTGTCACCGTCCAAAAAAGGTTTCAGCTACTGTTGAAGATGCTGATTCAATTTTGTGGTACC

TTCTTTGAATCGGTGCTGTTTGGTTCAGGGGATGGGATTGGGAGTGGTTCGATCGCATTCATTGATTCC

TCCCCTCTTTCTTTCTGTTATTGTCATTTTTGTTTTATTTTTTTTATTATTATTTTGGGTTAGAGTGATG

- $\mathrm{t}$--

TAGCTGCATGTCTTTTCTTITgGgTTGTTGAgGCTTATATACACTTATTAAGAAAGAAATCCATCATCAC

TATTAATAATCTCAACAACTCTTATCTCTCTCACATAAGTCACAACCCCCCTTTTTCCGT TTTTGTTACT

GCACACTTCATTGTTTGTTTGGTGGTGAACAATGAACATGCGACTAAACAAAATTCAAGAA

TGTCCGCACACTTCATTGTTTGTTTGGTGGGTGAACAATGAACATGCGACTAAAACAAAATTTCAAGAAA

AAGGAATACTTGTAGTTGAAGTGTTTAACAAATTAAATTTGATTCATTAGTTTAACTATTTTTTGTTGCA

-

CTTGTTTTTCCCACCACCCGTTTTCAATATTCTTGAACAAGTTTGTTGGTTTTGTTAGCCTATGTTTTTG

GTCCCCATAGTTTGGAACTCATTTACTTTTCTGTTGAATTTTITTATTGAGATGCAGTCTTTTCAATTCT

TCTCCCCTTTTTCAACATAAAACTAAACTTTTATTTATATTTATATGTGGATTTAGCTAAAGAGTATCCT --- g--_--AATGAAAGTTGAAAGTTTTGGTTCTTTCATGCATAGAAAACTTGAAAAATTTTAATTTACATTTTCTATT

CGAACTTTTTCTACTTGTGATGTCATTAAAggGCATtTGTTAgGCTTTCTCAGTCATGCATATGAACTTA -a--- c---ACAATCATGCATGTGTATCTTTTACTTT. ......... TCTATTTCTCCTTCTGATTTGGTACTCTGTTTT TTTCTCCTTGTTTGGGTATTGATTTTGGATCTCTTGATACTTTCTGCGTAATATTGTATGATTTTGATCA TTTCTCCTTGTTTGGGTATTGATTTTGGATCTCTTGATACTTTCTGCGTAATATTGTATGATTTTGATCA ATGCTGTGAACCGATGAGTGTGAATTATCATGCATAGGTGACTTGGGACTTTTTTGAAGTTTTGGTAACT ATGTTGATTATGTTGTATGAAACTGCTTTTGGGTCTTTACTGTGTCAAAGGTATTTTGCTGGCTCTTGTT ATGTTGATTATGTTGTATGAAACTGCTTTTGGGTOTTTACTGTGTCAAAGGTATTTTGCTGGCTCTTGTT GTGGTGATTTGAGTAACCTAGGCATGTGTTGGTTGCATGCTCTGCAAACCTGTTCTTGCTATGATTATTC TITGTAATTCTCTTAACATAATAGTGGATATgGCCTTTTTTTTTCT. . CTTATTTTTGTTGCCGTCAATA ATATGATCTTCGACAATTGATTTATTGTATTAGCATGGTAGGATCATTGTAGAATTATTAATCCGACATT ATATGATCTTCGACAATTGATTTATTGTATTAGCATGGTAGGATCATTGTAGAATTATTAATCCGACATT CATGTGCTTTTCTTTTGTAGAGAGGTTGAAAATCTCAAGAAGCCTTTCACGCCTCCCCGAGAGGTACATG ITCAAGTAACCCACTCCATGCCACCCCAGAAGATTGAGATTTTCAAATCGTTAGAGGGTTGGGCTGAGGA TTCAAGTAACCCACTCCATGCCACCCCAGAAGATT GAGATTTTCAAATCGTTAGAGGGTTGGGCTGAGGA GAACATTTTGACTCTCCTCAAACCTGTAGAGAAGTGCTGGCAGCCACAGGACTATTTACCAGAACCTTCA GAGGATGGATTTGAGGAGCAAGTGAGGGACTGAGAGCAAGGGCAAAAGAGCTTCCTGATGATTACTTTG GAGGATGGATTTGAGGAGCAAGTGAGGGAACTGAGAGCAAGGGCAAAAGAGCTTCCTGATGATTACTTTG ITGTTCTGGTCGGAGACATGATCACTGAAGAAGCCCTTCCTACTTACCAGACAATGCTTAATACTCTCGA CGGGGTTCGAGATGAAACAGGTGCCAGCCTTACTTCCTGGGCAGTTTGGACAAGGGCATGGACTGCGGAG CGGGGTTCGAGATGAAACAGGTGCCAGCCTTACTTCCTGGGCAGTTTGGACAAGGGCATGGACTGCGGAG GAAAATAGACATGGTGATCTTCTTAACAAGTATCTTTACTTGTCTGGCCGCGTTGACTTGAGGCAAATTG AGAAGACAATTCAGTACTTGATTGGCTCTGGAATGGTAAGATCTTATTGAATCTAGTAGTATGGTTATCT TAAGGTAGTATAGTAGATTTCTTTATGAATTTGCATAATCTTGCACCTGCTGAAAAGCCATTCAGACACT TAAGGTAGTATAGTAGATTTCTTTATGAATTTGCATAATCTTGCACCTGCTGAAAAGCCATTCAGACACT AATATATGTCACATTCTGgGTTCTTCAAATACACATATACATAGGCATCTCTAAACTATTGAAGGCACAC
70 70

140

137

210

207

280

277

350

347

420

417

490

487

560

556

630

626

700

696

770

766

840

836

910

906

980

976

1050

1046

1120

1116

1190

1186

1251

1256

1321

1326

1391

1395

1461

1465

1531

1535

1599

1605

1669

1675

1739

1745

1809

1815

1879

1885

1949

1955

2019

2025

2089

2095

2159

2165

2229

2235

2299

2305

2369 


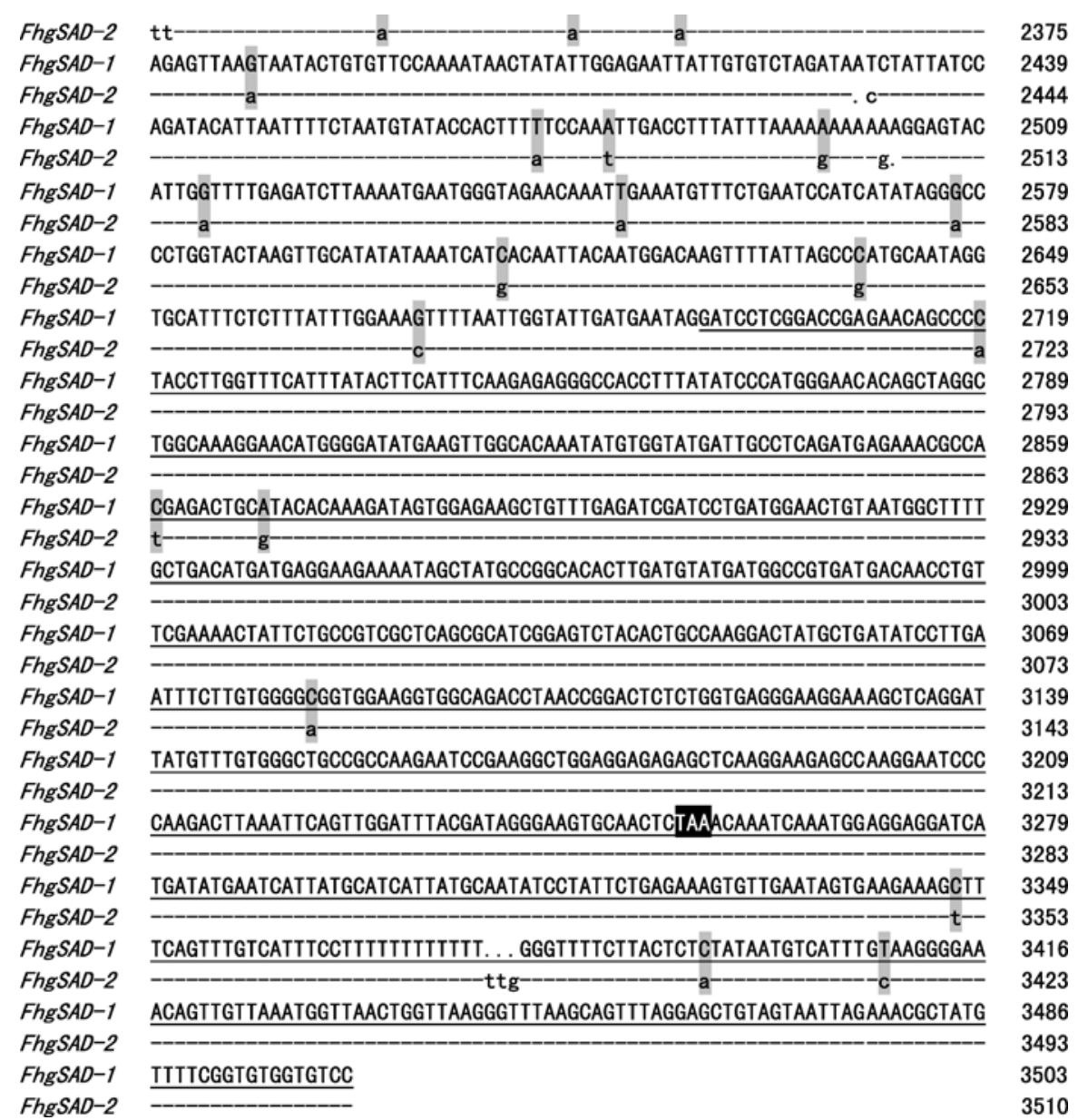

图 1 丰花 2 号 2 条 $S A D$ 核苷酸序列比对分析

Fig. 1 Alignment of two genomic nucleotide sequences of $S A D$ from Fenghua 2

下画线标注外显子部分; “.”表示核苷酸缺失; 黑色阴影标注起始密码子 ATG 和终止密码子 TAA; 灰色阴影标注 SNP 位点。 Exon is underlined; “"”indicates nucleotide deletion; initiator codon ATG and the stop codon TAA are shaded by dark blocks, the sites of SNP are shaded by gray.

基 ACP 脱氢酶家族和属于类铁蛋白家族的保守结 构域。位于 SAD 保守区的 4 个螺旋结构中埋藏着一 个由 2 个铁原子组成的二铁中心, $\mathrm{Fe}$ 原子的 6 个配 基即是四螺旋束的 6 个氨基酸的侧链或其基团：其 中一个铁原子的配基是 E239、H285, 另一个铁的配 基是 E148、H189，而 E186、E282 是 2 个铁原子的 桥连配基。两铁原子之间通过一个氧原子相联, 形 成非常对称的 $\mathrm{Fe}-\mathrm{O}-\mathrm{Fe}$ 二铁氧簇结构。SAD 每个亚 基表面有 33 个多肽连接位点。推断的底物结合位点 包括 14 个氨基酸的侧链或其基团(图 3 和图 5)。此 二铁中心及四螺旋束的氨基酸侧链形成 SAD 活性 中心。

2.43 个栽培品种和 2 个野生种间 $S A D$ 核苷酸序 列分析

以来自 3 个栽培品种的 FhgSAD-1、FhgSAD-2、 ShgSAD-1、ShgSAD-2、PygSAD-1、PygSAD-2 以及
来自野生种的 $g S A D-A$ 和 $g S A D-B$ 构建的进化树表明, FhgSAD-1、ShgSAD-1、PygSAD-1 和 $g S A D-A$ 聚为一 组, FhgSAD-2、ShgSAD-2、PygSAD-2 和 $g S A D-B$ 聚 为一组(图 4)。序列比对分析显示, FhgSAD-1 和 ShgSAD-1 序列完全相同, FhgSAD-2、ShgSAD-2、 $P y g S A D-2$ 和 $g S A D-B$ 完全相同。而 FhgSAD-1、 PygSAD-1 和 $g S A D-A$ 共存在 4 个碱基差异, 均在内 含子部分, 其中在第 2319 、第 2622 个碱基处属于 转换, 第 310 个碱基处属于颠换, 第 3377 个碱基处 属于缺失(表 2)。

2.5 花生与其他油料作物 $S A D$ 编码的氨基酸序 列分析

将 Ah-SAD1、Ah-SAD2、Ah-SAD3 与 NCBI 蛋白数据库中注册的其他油料作物 $S A D$ 编码的氨基 酸序列比对, 结果显示花生 Ah-SAD1 与大豆、麻风 树、芝麻、油茶、向日葵、甘蓝型油菜、拟南芥、 
(a) FhgSAD-1 CGGTGCTGtTtGgtTCAgGGGATtGGGATtGGGAGTGtTCGATCGCATTCATtGATtCC 490

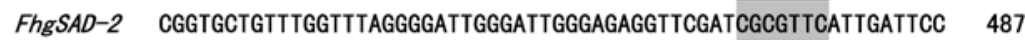

(b) FhgSAD-1 CTACTTGTGATGTCATTAAAGGCATTTGTTAGGCTTTCTCAGTCATGCATATGAACTTA 1190

FhgSAD-2 CTACTTCTGATGTCACTAAAGGGCATTTGTTAGGCTTTCTCAGTCATGCATAGGAACTTA 1186

(c) FhgSAD-1 ATATAGGGCCCCTGGTACTAAGTTGCATATATAAATCATCACAATTACAATGGACAAGTT 2639

FhgSAD-2 ATATAGGACCCCTGGTACTAAGTTGCATATATAAATCATGACAATTACAATGGACAAGTT 2643

(d) FhgSAD-1 CATtATGCATCATTATGCAATATCCTATTCTGAGAAAGTGTTGAATAGTGAAGAAAGCTT 3349

FhgSAD-2 CATTATGCATCATTATGCAATATCCTATTCTGAGAAAGTGTTGAATAGTGAAGAAAGTIT 3353

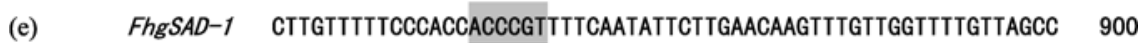

FhgSAD-2 CITGTTTTTCCCACCACCGGTTTTCAATATTCTTGAACAAGTTTGTTGGTTTGTTAGCC 896

(f)

FhgSAD-1 GAAAATAGACATGGTGATCTTCTTAACAAGTATCTTTACTTGTCTGGCCGCGTTGACTTG 2149

FhgSAD-2 GAAAATAGACACGGTGACCTTCTTAACAAGTATCTTTACTTGTCTGGCCGCGTTGACTTG 2155

图 2 FhgSAD-1 和 FhgSAD-2 部分核苷酸序列比对分析

Fig. 2 Alignment of partial genomic nucleotide sequences of FhgSAD-1 and FhgSAD-2

a: 黑色阴影所示序列是 Bsm I 可识别的核苷酸序列; 灰色阴影所示序列是 Bsm I 不能识别的核苷酸序列; b: 黑色阴影所示序列是 Nde I 可识别的核甘酸序列; 灰色阴影所示序列是 Nde I 不能识别的核甘酸序列; c: 黑色阴影所示序列是 Apa I 可识别的核苷酸序列; 灰色阴 影所示序列是 Apa I 不能识别的核苷酸序列; d: 黑色阴影所示序列是 Hind III 可识别的核苷酸序列; 灰色阴影所示序列是 Hind III 不 能识别的核苷酸序列; e: 黑色阴影所示序列是 Age I 可识别的核苷酸序列; 灰色阴影所示序列是 Age I 不能识别的核苷酸序列; f: 黑色

阴影所示序列是 BstE II 可识别的核苷酸序列; 灰色阴影所示序列是 BstE II 不能识别的核苷酸序列。

a: endonuclease recognition site of Bsm I is shaded by dark blocks, the unrecognitive nucleotides by gray; b: endonuclease recognition site of Nde I is shaded by dark blocks, the unrecognitive nucleotides by pale; c: endonuclease recognition site of Apa I is shaded by dark blocks, the unrecognitive nucleotides by gray; d: endonuclease recognition site of Hind III is shaded by dark blocks, the unrecognitive nucleotides by gray; e: endonuclease recognition site of Age I is shaded by dark blocks, the unrecognitive nucleotides by gray; f: endonuclease recognition site of BstE II is shaded by dark blocks, the unrecognitive nucleotides by gray.

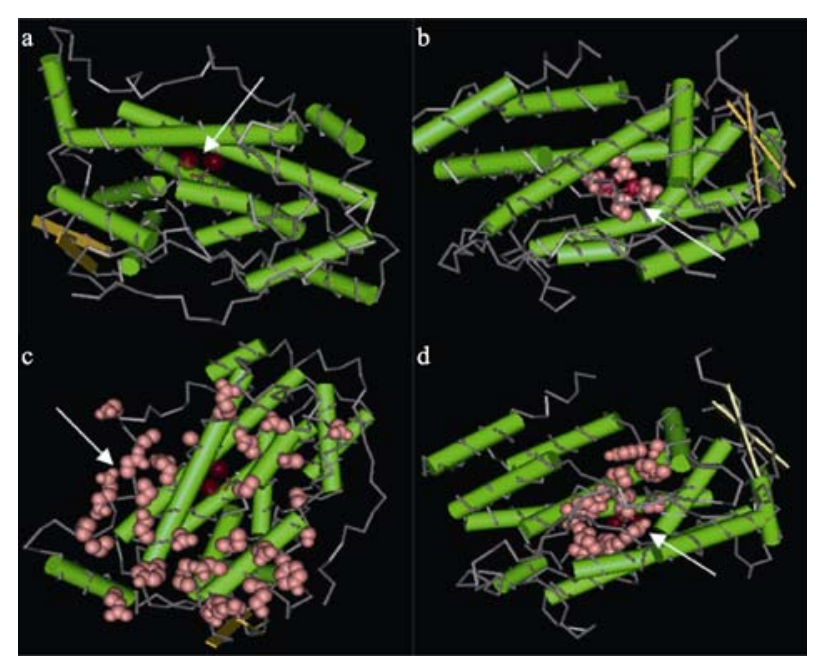

图 3 花生 Ah-SAD 理论三维结构

Fig. 3 Theoretical three-dimensional structure of Ah-SAD $\mathrm{a}: \mathrm{SAD}$ 保守区的二铁中心; b: Fe 原子的 6 个配基; $\mathrm{c}$ ：亚基表面的 多肽连接位点; d: 推断的底物结合位点。

a: diiron center on conserved domain of SAD; b: six bidentate ligand of Fe; c: polypeptide binding site; d: putative substrate binding pocket.

油橄榄和蓄麻间 SAD 氨基酸序列同源性依次为 $88.0 \% 、 85.2 \% 、 83.0 \% 、 82.8 \% 、 80.7 \% 、 80.2 \% 、 77.0 \%$ 、

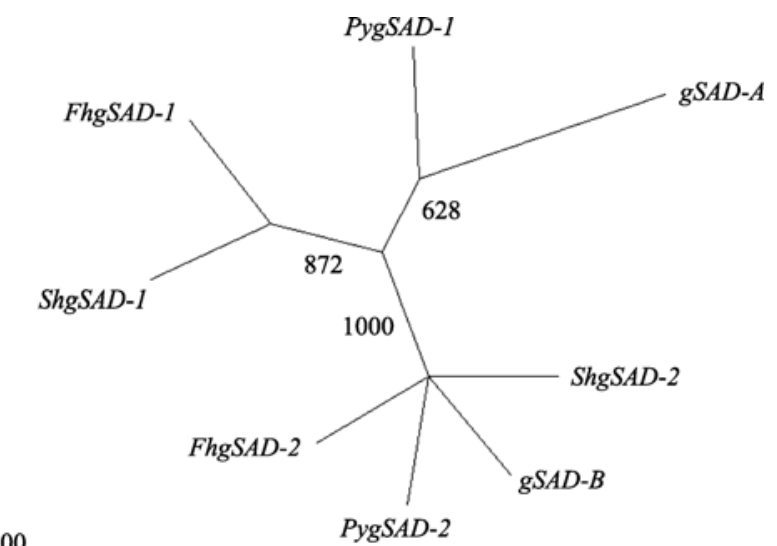

图 4 花生栽培品种和野生种 $S A D$ 分子进化树

Fig. 4 Phylogenetic tree based on the nucleotide sequence of $S A D$ genes from the cultivated peanut and wild diploid

$75.8 \%$ 和 $63.6 \%$ 。SAD 在不同物种间的同源性很高, 所有 SAD 的核心区段几乎完全相同，仅在起始密码 子和终止密码子附近有较大的差异(图 5)。聚类分析 表明，花生 SAD 与大豆的亲缘关系最近，并与麻风 树、油茶和芝麻聚为一类，又与向日葵聚为一大类; 油菜、拟南芥聚为一类, 油橄榄和蓄麻聚为一类。 这与各物种在经典分类学上的地位相一致(图 6)。 
表 2 不同花生品种中 $S A D$ 多态性位点

Table 2 Polymorphism sites of $S A D$ in different peanut cultivars

\begin{tabular}{ccccc}
\hline $\begin{array}{c}\text { 碱基位置 } \\
\text { Nucleotide site }\end{array}$ & FhgSAD-1 & ShgSAD-1 & PygSAD-1 & gSAD-A \\
\hline 310 & A & A & T & T \\
2319 & G & G & A & A \\
2622 & G & G & G & A \\
3377 & T & T & - & - \\
\hline
\end{tabular}

\section{3 讨论}

SAD 是高等植物中脂肪酸合成代谢的关键酶, 目前已从多种植物中分离获得 $S A D$ 的 $\mathrm{cDNA}$ 。其中 油菜、芝麻、油茶、麻风树等大多数油料作物 $S A D$ 基因的 ORF 长 1 173 1 $197 \mathrm{bp}$, 编码 390 398 个氨 基酸 ${ }^{[10,18-19]}$ 。本研究获得的 2 个花生 $S A D$ 基因全长 分别为 $3503 \mathrm{bp} 、 3510 \mathrm{bp}$, 均包括 3 个外显子和 2

\begin{tabular}{|c|c|}
\hline Ah-SAD1 & MALRLNPNPSQKLFLSPSSSSSSSSSSFSLPQMASLRSPRFRMAST-----LRTGSKEVEN 56 \\
\hline Ah-SAD3 & MALRLNPNPSQKLFLSPSSSSSSSSSSFSLPQMASLRSPRFRMAST----LRTGSKEVEN 56 \\
\hline Ah-SAD2 & MALRLNPNPSQKLFLSPSSSSSSSSS-FSLPQMASLRSPRFRMAST----LRTGSKEVEN 55 \\
\hline $\mathrm{Gm}$ & MALRLNPIPTQ----_-_-_----TFSLPQMASLRSPRFRMAST----'LRSGSKEVEN \\
\hline Si & MALKLNAINFQ--_-_-SPKCPSFALPPVASVRSPKFFMAST----LRSGSKEVET 46 \\
\hline Co & MAMKLNPNTFQ--_-_-YQKYPSFALPPMASLRSPKFFMAST----LRSGSKEVEN 46 \\
\hline Jc & MALKLNPF ISQ--_-_-_FHKLPTFALPPMANLRSPKFYMAST----LKSGSKEVEN 46 \\
\hline $\mathrm{Ha}$ & MAIRINTATFQ-------SDLYRSFAFPQPKPLRSPKFAMAST----IGSATTKVES \\
\hline At & MALKFNPLVAS--_-_QPYKFPSSTRPPTSSFRSPKFLCLASSSPALSSGPKEVES 51 \\
\hline Bn & MALKLNPLAS---_--QPYNFPSSARPPISTFRSPKFLCLASSSPALSS--KEVES 48 \\
\hline 0 e & MALKLCFPPHK--_-_MPSFPDARIRSHRVFMAST----IHSPSMEVGK \\
\hline Rc & $\begin{array}{l}\text { MQASHCLSLSL----_-SSHHNFTWTQHHHSLQPPQLRRLKP---- } \\
* \quad . . \quad \therefore .\end{array}$ \\
\hline
\end{tabular}

Ah-SAD1 LKKPFTPPREVHVQVTHSMPPQKIEIFKSLEGWAEENILTLLKPVEKCWQPQDYLPEPSE 116 Ah-SAD3 LKKPFTPPREVHVQGNHSMPPQKIEIFKSLEGWAEENILTLLKPVEKCWQPQDYLPEPSE 116 Ah-SAD2 LKKPFTPPREVHVQVTHSMPPQKIEIFKSLEGWAEENILTLLKPVEKCWQPQDYLPEPSE 115 IKKPFTPPREVHVQVTHSMPPQKIEIFQSLEDWAEENILAHLKPVEKCWQPQDFLPDPSS 101 VKRPFNPPREVHVQVTHSMPPQKIEIFKALEDWADNNILVHLKPVEKCWQPQDFLPDPSS 106 LRKPFSPPREVHVQVTHSMPPQKIEIFKNLEDWADQNILVHRLPVEKCWQPQDFLPDPAS 106 LKKPFMPPREVHVQVTHSMPPQKIEIFKSLDEWAEQN ILVHLKPVEKCWQPQDFLPDPSS 106 TKKPFTPPREVHQQVLHSMPPQKIEIFKSMEGWAENNILVHLKPVEKCWQAQDFLPDPAS 106 LKKPFTPPREVHVQVLHSMPPQKIEIFKSMENWAEENLLIHLKDVEKSWQPQDFLPDPAS 111 LKKPFTPPKEVHVQVLHSMPPQKIEIFKSMEDWAEQNLLTQLKDVEKSWQPQDFLPDPAS 108 VKKPFTPPREVHVQVTHSLAPEKREIFNSLNNWAQENILVLLKDVDKCWQPSDFLPDSAS 100 VAAPAQPLK--HQTKTHSMPQEKIEIFKSLENWATQEVLPLLKPVDKCWQPQNFLPDPAL 97

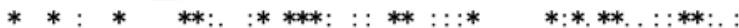

Ah-SAD1 -DGFEEQVRELRARAKELPDDYFVVLVGDMITEEALPTYOTMLNTLDGVRDETGASLTS 174 Ah-SAD3 --DGFEEQVRELRARAKELPDDYFVVVGDMI TEEALPTYQTMLNTLDGVRDETGASLTS 174 Ah-SAD2 --DGFEEQVRELRARAKELPDDYFVVLVGDMITEEALPTYQTMLNTLDGVRDETGASLTS 173 $\mathrm{Gm}$ --DGFEEQVKELRERAKELPDDYFVVLVGDMITEEALPTYQTMLNTLDGVRDETGASLTS 159 -DGFDDQVKELRERAKEIPDDYFVVLVGDMITEEALPTYQTMLNTLDGVRDETGASPTS 164 --EGFDEQVRELRERAKEIPDDYFVVLVGDM I TEEALPTYQTMLNTLDGVRDETGASLTS 164 --DGFDEQVRELRERVKEI PDDYFVVLVGDMITEEALPTYQTMLNTLDGVRDETGASLTS 164 --DGFMEQVEELRARAKEIPDDYFVVLVGDM ITEEALPTYQTMLNTLDGVRDETGATLLL 164 -DGFEDQVRELRERARDLPDDYFVVLVGDMI TEEALPTYQTMLNTLDGVRDETGASPTS 169 --DGFEDQVRELRERARELPDDYFVVLVGDMITEEALPTYQTMLNTLDGVRDETGASPTS 166 --EGFDEQVMELRKRCKE IPDDYF IVLVGDM I TEEALPTYQTMLNTLDGVRDETGASLTP 158 PFDEFTDQVRALRDRTAELPDEYFVVLVGDMITEDALPTYQTMINTLDGVKDETGASNNP 157

$: *: * * \quad * * * \quad:: * *: * *: * * * * * * * * * * ; * * * * * * * * * ; * * * * * *: * * * * *:$

WAVWTRAWTAEENRHGDLLNKYLYLSGRVDLRQIEKT I QYL I GSGMDPRTENSPYLGF I 234 WAVWTRAWTAEENRHGDLLNKYLYLSGRVDLRQIEKT I QYL IGSGMDPRTENSPYLGF IY 234 WAVWTRAWTAEENRHGDLLNKYLYLSGRVDLRQIEKT I QYLIGSGMDPRTENSPYLGF IY 233 WA IWTRAWTAEENRHGDLLNKYLYLSGRVDMKQIEKT I QYLI IGSGMDPRTENSPYLGF IY 219 WAIWTRAWTAEENRHGDLLNKYLYLSGRVDMRQIEKT I QYL I GSGMDPRTENSPYLGF IY 224 WAVWTRAWTAEENRHGDLLNKYLYLSGRVDMRQIEKT I QYL I GSGMDPRTENSPYLGF IY 224 WAIWTRAWTAEENRHGDLLNKYLYLSGRVDMRQIEKT I QYLI GSGMDPRTENSPYLGF IY 224 GLVWTRAWTAEENRHGDLLHQYLYLSGRVDMRQI QKT I QYLI GSGMDPRTENSPYLGF IY 224 WAIWTRAWTAEENAHGDLLNKYLYLSGRVDMRQIEKT I QYLIGSGMDPRTENNPYLGF IY 229 WAI WTRAWTAEENRHGDLLNKYLYLSGRVDMRQIEKT I QYLI GSGMDPRTENNPYLGF IY 226 WAIWTRAWTAEENRHGDLLNKYLYLSGRVDMKQIEKT IQYLIGSGMDPRTENNPYLGF IY 218 WALWTRSWTAEENRHGDLLRSFLYLSGRVDMLMIEKTVQYLI GAGMDPGTENNPYLGYVY 217 
Ah-SAD1 ISFQERATF ISHGNTARLAKEHGDMKLAQICGMIASDEKRHETAYTK IVEKLFE IDPDGT 294 Ah-SAD3 TSFQERATF I SHGNTARLAKGHGDMKLAQI CGM I ASDEKRHETAYTK IVEKLFEIDPDGT 294 Ah-SAD2 TSFQERATF ISHGNTARLAKEHGDMKLAQICGMIASDEKRHETAYTK IVEKLFEIDPDGT 293 Gm TSFQERATF ISHGNTARLAKEHGDIKLAQI GGM I ASDEKRHETAYTK IVEKLFESDPDGT 279 $\mathrm{Si}$ TSFQERATF ISHGNTARLAREHGDLKLAQI IGT I AADEKRHETAYTK IVEKLFE IDPNDT 284 Co TSFQERATF I SHGNTARLAKEHGD I KLAQICGT I ASDEKRHETAYTK IVEKLFE IDPDGT 284 Jc TSFQERATFISHGNTARLAKEHGDIKLAQICGT I AADEKRHETAYTK IVEKLFE IDPDGT 284 Ha TSFQERATF I SHGNTARHAKEHGDVKLAQMCG I IAADEKRHETAYTK IVEKLFE IDPDGT 284 At TSFQERATF I SHGNTARQAKEHGDIKLAQICGT IAADEKRHETAYTK IVEKLFE IDPDGT 289 Bn TSFQERATFISHGNTARQAKEHGDLKLAQICGT IAADEKRHETAYTK IVEKLFEIDPDGT 286 Oe TSFQERATF I SHGNTARLAKEHGDLKLAQICG I IAADEKRHETAYTK IVEKLFE IDPDGT 278 Rc TSFQERATFVSHGNTARLAKEGGDPILARI CGT IASDEKRHENAYAR IVEKLLEVDPTGA 277 $* * * * * * * * * * * * * * * * * * * * ;: * * * * *: ; * * * *: * * * * * * *, * *:: * * * * * * * * * *$.:

Ah-SAD1 VMAFADMMRKKIAMPAHLMYDGRDDNLFNYSAVAQRI GVYTAKDYADILEFLVGRWKVA 354 Ah-SAD3 VMAFADMMRKKIAMPAHLMYDGRDDNLFENYSAVAQRI GVYTAKDYADILEFLVARWKVA 354 Ah-SAD2 VMAFADMMRKKIAMPAHLMYDGRDDNLFENYSAVAQRIGVYTAKDYADILEFLVGRWKVA 353 $\mathrm{Gm}$ VMAFADMMRKKI AMPAHLMYDGRDDNLFDNYSSVAQRIGVYTAKDYADILEFLVGRWKVE 339 VLAFADMMRKK I SMPAHLMYDGRDDNLFDHFSSVAQRLGVYTAKDYADILEHLVARWKVA 344 VLALADMMRKKI SMPAHLMYDGRDDNLFEHFSSVAQRLGVYTAKDYADILEFLVGRWQVE 344 VLAFADMMRKKI SMPAHLMYDGRDDNLFDHFSAVAQRLGVYTAKDYADILEFLVGRWKVD 344 VLAFADMMRKKI SMPAHLMYDGRDDNLFENFSAVAQRLGVYTAKDYADILEFLVGRWKVA 344 VMAFADMMRKKI SMPAHLMYDGANDNLFDNFSSVAQRLGVYTAKDYADILEFLVGRWKIQ 349 VMAFADMMRKK I SMPAHLMYDGRDESLFDNFSSVAQRLGVYTAKDYADILEFLVGRWKIE 346 VLALADMMRKKVSMPAHLMYDGQDDNLFENFSSVAQRLGVYTAKDYADILEFLVGRWDIE 338 MVAI ADMMHKKI TMPAHLMYDGQDPQLFQHFSAVAQRLGVYTADDYADILEFLIGRWRLE 337

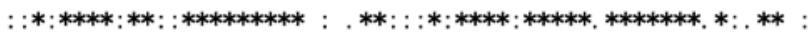

DLTGLSGEGRKAQDYVCGLPPRIR---RLEERAQGRAK---ESPRLKFSWIYDREVQL 406 DLTGLSGEGRKAQDYVCRCRPRIRRIRRLEERAQGRAK----ESPRLKFSWI YDREVQL 409 DLTGLSGEGRKAQDYVCGLPPRIR---RLEERAQGRAK----ESPRLKFSWIYDREVQL 405 QLTGLSGEGRKAQEYICGLPPRIR---RLEERAQARVK----ESSTLKFSWIHDRESTT 391 NLTGLSADGRKAQDYVCGLPPRIR---RLEERAQGRAK----QAPK IPFSWI HDREVQL 396 ELTGLSAEGRKAQDYVCGLPPRIR---KLEERAQSRVK----QVRPVPFSWIFDRELKI 396 KLTGLSAEGQKAQDYVCRLPPRIR---RLEERAQGRAK----EGPT IPFSWIFDREVKL 396 DLTGLSGEGRKAQDYVCGLAPRIR---RLEERNSARAK----ESVNVPFSWIFDREVKL 396 DLTGLSGEGNKAQDYLCGLAPRIK---RLDERAQARAKLKGQROKPILPVPHF ICLLLKL 406 SLTGLSGEGNKAQEYLCGLTPRIR---RLDERAQARAK-KG----PKVPFSWIHDREVQL 398 KLTGLSGEGRKAQDYVCTLPPRIR---RLEERAQSRVK----KASATPFSWIFGRE INL 390 KLEGMTGEGRRAQDFVGGLAPRIR--KLQERADERAK---KMKPQSAKFSWIFNREVPL 391

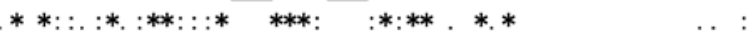

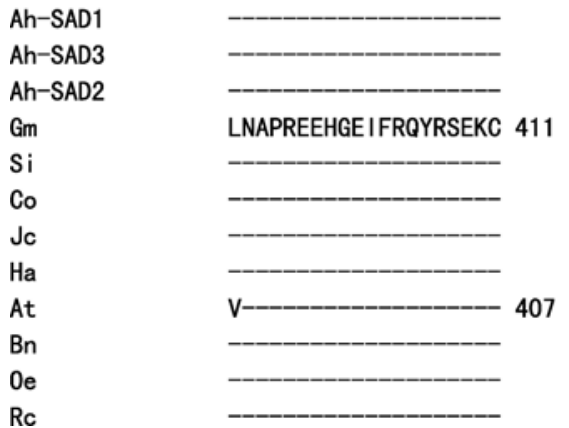

图 5 花生与主要油料作物 $S A D$ 编码的氨基酸序列比较

Fig. 5 Alignment of $S A D$ amino acid sequences of peanut and other major oil plants

$\mathrm{Ah}$ : 花生; $\mathrm{Gm}$ : 大豆; $\mathrm{Si}$ : 芝麻; Co: 油茶; Jc: 麻风树; Ha: 向日葵; At: 拟南芥; Bn: 甘蓝型油菜; Oe: 油橄榄; Rc: 菎麻。“一”表示 该处氨基酸残基缺失; 灰色阴影标注 Ah-SAD1 和 Ah-SAD3 的氨基酸差异; 黑色阴影标注 14 个底物结合位点; “ *”表示该处氨基酸 完全相同。

Ah: Arachis hypogae; Gm: Glycine max; Si: Sesamum indicum; Co: Camellia oleifera; Jc: Jatropha curcas; At: Arabidopsis thaliana; Bn: Brassica napus; Ha: Helianthus annuus; Oe: Olea europae; Rc: Ricinus communis. "_-" represents absences of amino acids residual, the difference amino acids between Ah-SAD1 and Ah-SAD3 are shaded by gray blocks; the sites of substrate binding pocket variations by dark. "*" indicates that the amino acids are identical. 


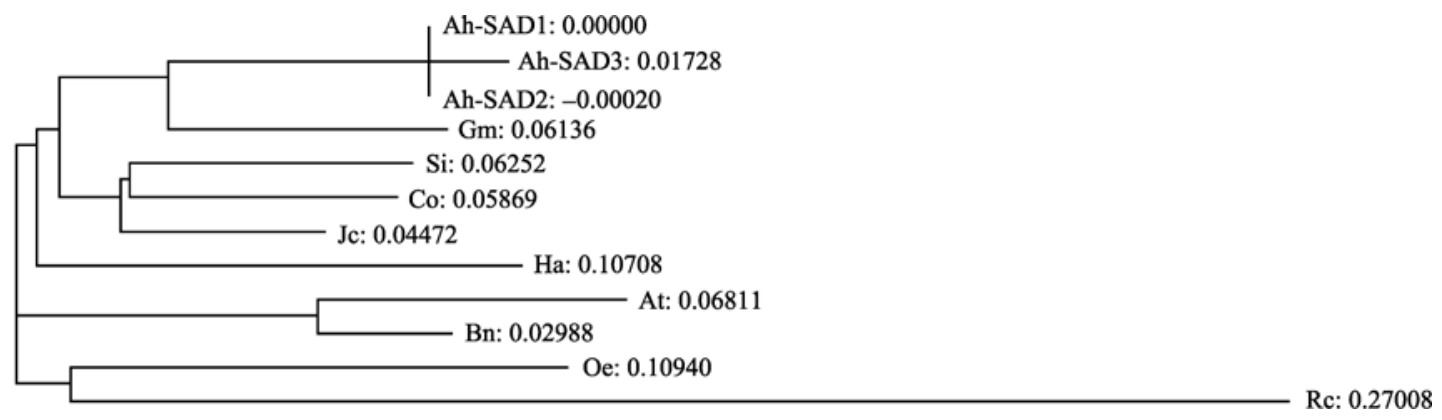

图 6 花生与 9 种主要油料作物 SAD 氨基酸序列的聚类分析

Fig. 6 Clustering of Ah-SAD and other SAD of nine oil plants based on amino acid sequence

$\mathrm{Ah}$ : 花生; $\mathrm{Gm}$ : 大豆; $\mathrm{Si}$ : 芝麻; Co：油茶; Jc：麻风树; Ha: 向日葵; At：拟南芥; Bn：甘蓝型油菜; Oe: 油橄榄; Rc: 蓖麻。

Ah: Arachis hypogae; Gm: Glycine max; Si: Sesamum indicum; Co: Camellia oleifera; Jc: Jatropha curcas; At: Arabidopsis thaliana; Bn: Brassica napus; Ha: Helianthus annuus; Oe: Olea europae; Rc: Ricinus communis.

个内含子, 其编码区长 $1221 \mathrm{bp} 、 1218 \mathrm{bp}$, 分别编 码 406 个、405 个氨基酸。通过与其他植物 SAD 氨 基酸序列比对可以发现, 花生 $\mathrm{SAD}$ 氨基酸序列 $\mathrm{N}$ 端 存在一段特有的 ${ }^{17}$ PSSSSSSSSSSFSL ${ }^{30}$ 丝氨酸聚集 区, 并且 Ah-SAD2 比 Ah-SAD1 少一个丝氨酸。董 胜张等 ${ }^{[20]}$ 和 Sappington 等 ${ }^{[21]}$ 研究发现大多数昆虫的 卵黄原蛋白 $(\mathrm{Vg})$ 氨基酸序列中存在一段丝氨酸聚集 域, 通常位于进化速率较高的区域, 可能是某些激 酶(如磷酸化激酶)的底物。对于花生 SAD 非保守的 丝氨酸聚集区的作用有待进一步研究。

Lindqvist 等 ${ }^{[2]}$ 用 $\mathrm{X}$-射线晶体衍射分析描述了 蓄麻 SAD 的晶体结构, 张党权等 ${ }^{[19]}$ 通过同源建模法 获得油茶 SAD 的理论三维结构, 均为同型二聚体, 亚基含有 11 个 $\alpha$-螺旋, 其中 9 个 $\alpha$-螺旋形成一个反 平行螺旋束, 该螺旋束上的 4 个 $\alpha$-螺旋与 $\mathrm{Fe}^{2+}$ 共同 形成 SAD 的活性中心。本研究获得的花生 SAD 存 在同型二聚体亚基结合位点和底物作用位点, 具有 植物 SAD 的空间结构特征。这为进一步从蛋白水平 研究花生 SAD 的功能奠定了基础。

花生栽培种(Arachis hypogaea L.)是异源四倍体 (AABB)。花生区组除了栽培种和 A. monticola 是四 倍体外, 其他都是二倍体野生种 ${ }^{[23-24]}$ 。一般认为花 生栽培种是由 2 个具有不同染色体组的二倍体野生 种通过自然杂交形成的杂种再经一次性加倍事件进 化形成 ${ }^{[25]}$ 。探寻花生栽培种的二倍体野生种亲本一 直是有关学者的研究热点, Moretzsohn 等 ${ }^{[26]}$ 通过微 卫星分析, Seijo 等 ${ }^{[27]}$ 利用 GISH 技术, Milla 等 ${ }^{[28]}$ 采 用 AFLP 方法均推断, A 组野生种 A. duranensis 和 B 组野生种 A. ipaensis 为栽培种 A、B 2 个染色体组的 供体。Fávero 等 ${ }^{[29]}$ 成功构建了由二倍体种 A. duranensis 和 A. ipaensis 组成的双二倍体, 并利用所获得
的双二倍体分别与栽培种几大植物学类型种质杂交, 得到了杂种后代植株, 进一步证明 A. duranensis 和 A. ipaensis 可能是栽培种的祖先。Jung 等 ${ }^{[30]}$ 通过对 栽培种和野生种 $\Delta^{12}$-脂肪酸脱氢酶基因序列的研究, 发现 A. duranensis 和 A. ipaensis 与栽培种的 2 个非 等位基因 AhFAD2A 和 AhFAD2B 分别有相同的基因 序列, 从而推断这 2 个野生种最有可能是花生栽培 种的二倍体祖先种。本研究获得的 $g S A D-1$ 和 $g S A D-2$ 同源性 $97.5 \%$ 。利用栽培品种和野生种 $S A D$ 的核酸序列构建的系统进化树表明, 来自 3 个栽培 品种 $g S A D-1$ 序列与野生种 A. duranensis (AA)的 $g S A D-A$ 聚在一组, $g S A D-2$ 序列与 $A$. ipaensis (BB) 的 $S A D-B$ 序列聚在一组。来源于 $\mathrm{A}$ 染色体组的 4 条 DNA 序列(FhgSAD-1、ShgSAD-1、PygSAD-1 和 $g S A D-A)$ 存在等位变异, 但碱基差异位点均出现在 内含子区域, 并未导致编码氨基酸序列的改变。来 自 B 染色体组的 $S A D$ 非常保守, 3 个栽培品种 $g S A D-$ 2 序列与野生种 A. ipaensis (BB) 的 $g S A D-B$ 序列完全 相同。推断栽培品种 $g S A D-1$ 和 $g S A D-2$ 分别来自花 生 A、B 染色体组。

\section{4 结论}

利用同源克隆技术获得了花生区组 2 个二倍体 野生种和 3 个栽培品种的 $S A D$, 每个栽培品种有 2 个 $S A D$ 。同时获得丰花 2 号 $S A D$ 的 2 条全长 $\mathrm{cDNA}$ 。 丰花 2 号的 FhgSAD-1 和 FhgSAD-2 均含有 2 个内含 子, 二者变异位点丰富。FhrSAD-1 和 FhrSAD-2 间 核苷酸序列共有 12 个变异位点, 编码的 Ah-SAD2 氨基酸序列与 Ah-SAD1 相比在 $\mathrm{N}$ 端的 ${ }^{17} \mathrm{PSSSSS}$ $\operatorname{SSSSSFSL}^{30}$ 丝氨酸聚集区少一个丝氨酸。 $g S A D-1$ 和 $g S A D-A$ 同源性为 $99.9 \%, g S A D-2$ 和 $g S A D-B$ 同源 
性为 $100 \%$ 。推测 $g S A D-1$ 和 $g S A D-2$ 分别来自花生 栽培品种的 A、B 2 个染色体组。

\section{References}

[1] Wan S-B(万书波). Peanut Quality (花生品质学). Beijing: China Agricultural Science and Technology Press, 2005. pp 2-10 (in Chinese)

[2] Chu Y, Holbrook C C, Ozias-Akins P. Two alleles of ahFAD2B control the high oleic acid trait in cultivated peanut. Crop Sci, 2009, 49: 2029-2036

[3] Yukawa Y, Takaiwa F, Shojik K, Masuda K, Yamada K. Structure and expression of two seed-specific cDNA encoding stearoyl-acyl carrier protein desaturase from sesame, Sesamum indicum. Plant Cell Physiol, 1996, 37: 201-205

[4] Li X-D(李晓丹), Cao Y-L(曹应龙), Hu Y(胡亚), Xiao L(肖玲), Wu Y-H(武玉花), Wu G(吴刚), Lu C-M(卢长明). Fatty acid accumulation pattern in developing seeds of peanut. Chin J Oil Crop Sci (中国油料作物学报), 2009, 31(2): 157-162 (in Chinese with English abstract)

[5] Wendy C, Paolo L, Nunzia S, Monica D P, Paola S, Virginia C, Noreen M C, Alan M M, Peter M, Tony A K, Philip J D, Stefania G, Teodoro C. Transplastomic tobacco plants expressing a fatty acid desaturase gene exhibit altered fatty acid profiles and improved cold tolerance. Transgenic Res, 2008, 17: 769-782

[6] Kachroo A, Shanklin J, Whittle E, Lapchyk L, Hildebrand D, Kachroo P. The Arabidopsis stearoyl-acyl carrier proteindesaturase family and the contribution of leaf isoforms to oleic acid synthesis. Plant Mol Biol, 2007, 63: 257-271

[7] Byfield G E, Xue H, Upchurch R G. Two genes from soybean encoding soluble $\Delta^{9}$-stearoyl-ACP desaturase. Crop Sci, 2006, 46: $840-846$

[8] Ping Z, Joseph W B, Robert G U, Edward W, John S, Ralph E D. Mutations in a $\Delta^{9}$-stearoyl-ACP-desaturase gene are associated with enhanced stearic acid levels in soybean seeds. Crop Sci, 2008, 48: 2305-2313

[9] Aardra K, John S, Edward W, Ludmila L, David H, Pradeep K. The Arabidopsis stearoyl-acyl carrier protein-desaturase family and the contribution of leaf isoforms to oleic acid synthesis. Plant Mol Biol, 2007, 63: 257-271

[10] Luo T, Deng W Y, Zeng J, Zhang F I. Cloning and characterization of a stearoyl-acyl carrier protein desaturase gene from Cinnamomum longepaniculatum. Plant Mol Biol Rep, 2009, 27: $13-19$

[11] Florin S, Yael B, Arnon B, Ilan H, Ran H. Identification and molecular characterization of homeologous $\Delta^{9}$-stearoyl-acyl carrier protein desaturase3 genes from the allotetraploid peanut (Arachis hypogaea). Plant Mol Biol Rep, 2010, 29: 232-241

[12] Whittle E, Cahoon E B, Subrahmanyam S, Shanklin J. A multifunctional acyl-acyl carrier protein desaturase from Hedera helix L. (English ivy) can synthesize 16- and 18-carbon monoene and diene products. J Biol Chem, 2005, 280: 28169-28176

[13] Zaborowska Z, Starzycki M, Femiak I, Swiderski M, Legocki A B. Yellow lupine gene encoding stearoyl-ACP desaturaseorganization, expression and potential application. Acta Biochim Polonicn, 2002, 49: 29-42

[14] Chen M N, Ren Z K, Chi X Y, Pan L J, Yu S L, Yang Q L. Isolation, characterization and expression analysis of stearoyl-ACP desaturase gene from Kosteletzkya virginica. Bioinform Biomed Engin (iCBBE), 2010, 4: 1-5

[15] Shah F H, Rashid O, San C T. Temporal regulation of two isoforms of cDNA clones encoding delta 9-stearoyl-ACP desaturase from oil palm (Elaies guineensis). Plant Sci, 2000, 152: 27-33

[16] Liu Q, Singh S P, Green A G. High-steric and oleic cottonseed oils produced by hairpin RNA-mediated post-transcriptional gene silencing. Plant Physiol, 2002, 129: 1-12

[17] Wendy C, Paolo L, Nunzia S, Monica D P, Paola S, Virginia C, Noreen M C, Alan M M, Peter M, Tony A K, Philip J D, Stefania G, Teodoro C. Transplastomic tobacco plants expressing a fatty acid desaturase gene exhibit altered fatty acid profiles and improved cold tolerance. Transgenic Res, 2008, 17: 769-782

[18] Thompson G A, Scherer D E, Aken S F, Kenny J W, Young H L, Shintani D K, Kridl J C, Knauf V C. Primary structures of the precursor and mature forms of stearoyl-acyl carrier protein desaturase from safflower embryos and requirement of ferredoxin for enzyme activity. Proc Natl Acad Sci USA, 1991, 88: $2578-2582$

[19] Zhang D-Q(张党权), Tan X-F(谭晓风), Chen H-P(陈鸿鹏), Zeng Y-L(曾艳玲), Jiang Y(蒋瑶), Li W(李魏), Hu F-M(胡芳 名). Full-length cDNA cloning and bioinformatic analysis of Camellia oleifera SAD. Sci Silv Sin (林业科学), 2008, 44(2): 155-159 (in Chinese with English abstract)

[20] Dong S-Z(董胜张), Ye G-Y(叶恭银), Liu C-L(刘朝良). Research progress in molecular evolution of yolk proteins in insects. Acta Entomol Sin (昆虫学报), 2008，51(11)：1196-1209 (in Chinese with English abstract)

[21] Sappington T W, Raikhel A S. Molecular characteristics of insect vitellogenin and vitellogenin receptors. Insect Biochem Mol Biol, 1998, 28: 277-300

[22] Lindqvist Y, Huang W, Schneider G, Shanklin J. Crystal structure of delta9-stearoyl-acyl carrier protein desaturase from castor 
seed and its relationship to other diiron proteins. EMBO J, 1996,

15: 4081-4092

[23] Krapovickas A, Gregory W C. Taxonomia del género Arachis (Leguminosae). Bonplandia, 1994, 8: 1-186

[24] Valls J F M, Simpson C E: New species of Arachis from Brazil, Paraguay, and Bolivia. Bonplandia, 2005, 14: 35-64

[25] Smartt J, Gregory W C, Gregory M P. The genomes of Arachis hypogaea L. cytogenetic studies of putative genome donor. Euphytica, 1978, 27: 665-675

[26] Moretzsohn M C, Hopkins M S, Mitchell S E, Kresovich S, Valls J F M, Ferreira M E. Genetic diversity of peanut (Arachis hypogaea L.) and its wild relatives based on the analysis of hypervariable regions of the genome. BMC Plant Biol, 2004, 4: 11

[27] Seijo G, Lavia G I, Fernández A, Krapovickas A, Ducasse D A,
Bertioli D J, Moscone E A. Genomic relationships between the cultivated peanut (Arachis hypogaea Leguminosae) and its close relatives revealed by double GISH. Am J Bot, 2007, 94: 1963-1971

[28] Milla S R, Isleib T G, Stalker H T. Taxonomic relationships among Arachis sect. Arachis species as revealed by AFLP markers. Genome, 2005, 48: 1-11

[29] Fávero A P, Simpson C E, Valls J F M, Vello N A. Study of the evolution of cultivated peanut through crossability studies among Arachis ipaënsis, A. duranensis, and A. hypogaea. Crop Sci, 2006, 46: $1546-1552$

[30] Jung S, Tate P L, Horn R, Kochert G, Moore K, Abbott A G. The phylogenetic relationship of possible progenitors of the cultivated peanut. J Hered, 2003, 94: 334-340 